University of Konstanz

Department of Economics

\title{
On the Optimal Size of Committees of Experts
}

\author{
Volker Hahn
}

Working Paper Series

2012-24

http://www.wiwi.uni-konstanz.de/forschung/ 


\title{
On the Optimal Size of Committees of Experts*
}

\author{
Volker Hahn \\ Department of Economics \\ University of Konstanz \\ Box 143 \\ 78457 Konstanz, Germany \\ volker.hahn@uni-konstanz.de
}

First Version: July 2011

This Version: November 2012

\begin{abstract}
What is the optimal size of expert committees? To address this question, I present a model of a committee of experts with career concerns. Each expert may observe an argument about the state of the world but be unsure about the argument's soundness. Experts may remain silent or compete for the opportunity to announce an argument. I show that experts become more reluctant to speak in larger committees. This effect is sufficiently strong to make small groups of experts optimal. At the same time, a small committee may be superior to an individual expert.
\end{abstract}

Keywords: experts, committees, career concerns, verifiable information, information aggregation.

JEL: $\quad$ D71, D82.

*I would like to thank Tobias Fleischer and participants at the annual meeting of the Verein für Socialpolitik in Göttingen, 2012, for many valuable comments and suggestions. 
Silence is the highest wisdom of a fool as speech is the greatest trial of a wise man.

Sir Francis Quarles (1592-1644), English poet

\section{Introduction}

Expert committees are frequently consulted when complex decisions have to be taken. Examples of such committees include councils of economic advisers as well as other advisory committees to government authorities. In the United States, the Food and Drug Administration alone seeks expert advice from 50 committees and panels. ${ }^{1}$ Given the prevalence of advisory committees, it is important to ask how they should be designed, and in particular, how large they should be.

A naïve view inspired by the Condorcet Jury Theorem (Condorcet, 1785) would suggest that an advisory committee should comprise as many experts as possible provided the consequences of the decision at hand are far-reaching and potentially severe and the costs of hiring experts low in comparison. Seeking advice from all available experts would minimize the chances that some crucial piece of evidence is overlooked. In this paper, I show that, while larger committees indeed ensure a high probability that significant information is known to at least one member of the committee, this does not imply that large committees are always desirable because larger groups may make individual experts more reluctant to speak up.

To show this, I set up a model, populated by a principal and a group of experts interested in appearing as competent individuals. Experts privately observe arguments against the prevailing view regarding an issue to be decided by the principal but are uncertain whether the arguments will hold up to public scrutiny. When contemplating making their views public, the experts have to weigh the possible gain in prestige, should their arguments hold water, against the potential loss in reputation, should public debate reveal flaws in their arguments.

The potential loss in reputation can be severe enough to induce experts to practice self-censorship and to withhold information going against conventional wisdom. The

\footnotetext{
${ }^{1}$ See http://www.fda.gov/AdvisoryCommittees/default.htm. Retrieved 15 June 2012.
} 
relevance of self-censorship in groups is demonstrated in an influential book by Janis (1972). He studies a host of episodes like the Bay of Pigs Invasion under the Kennedy administration and argues that a phenomenon he labels "groupthink" can occur, which causes individuals to suppress arguments against the prevailing view.

In my model, the trade-off between the costs and benefits of speech is influenced by group size. An individual expert is more likely to be the first to raise an argument if it is far-fetched and hence not shared by many of his colleagues. As a result, the ability to present an argument first may in itself be an indication that this argument is inessential. This effect is powerful in large committees, where it is particularly unlikely that a substantive argument can be released by a particular expert ahead of his colleagues. As a consequence, experts on large committees will be reluctant to reveal their views. I show that this effect is strong enough to make small committees preferable both in comparison with large ones and a single expert.

The paper is organized as follows: The next section surveys the related literature. I present the model in Section 3. Results on the optimal behavior of experts, the equilibria and welfare are derived in Section 4. I discuss several extensions and the robustness of my findings in Section 5. Section 6 concludes.

\section{Related Literature}

In the present paper, I consider a committee of experts with private information about the state of the world. Thus, my paper is related to the vast literature on group decision making. ${ }^{2}$ One of the main theoretical justifications for delegating decision-making to a committee rather than an individual is the Condorcet Jury Theorem (Condorcet, 1785), according to which (i) the quality of committee decision-making increases monotonically with committee size and (ii) the probability of a correct decision becomes one if committee size goes to infinity. This theorem has been extended in various directions, e.g. varying voter competencies and dependent votes (see Boland (1989), Berg (1993), and Ladha (1992)). Austen-Smith and Banks (1996) criticize the assumption of sincere voting employed by the traditional literature on the Condorcet Jury Theorem

\footnotetext{
${ }^{2}$ Recent surveys of the literature on committee decision-making are Gerling et al. (2005), Li and Suen (2009), as well as Austen-Smith and Feddersen (2009).
} 
and show that the theorem may fail to hold in a game-theoretic framework. ${ }^{3}$ Feddersen and Pesendorfer (1997) as well as Wit (1998) prove that the Condorcet Jury Theorem survives if equilibria in mixed strategies are taken into account. In contrast to the bulk of this literature, I consider an advisory committee that delivers information to a principal who aggregates all information optimally rather than a committee whose members aggregate information through voting (see Ottaviani and Sørensen (2001), Visser and Swank (2007), and Hahn (2011) for analyses of debate among experts).

While the Condorcet Jury Theorem implies that committees should be as large as possible, some papers have identified a rationale for the optimality of small committees that differs from the one proposed here. If information acquisition is costly to individuals, there will be free-riding incentives, which are particularly severe for large committees (see Ben-Yashar and Nitzan (2001) and Mukhopadhaya (2003)). ${ }^{4}$ As a result, very large committees may deliver a lower quality of decision-making compared to smaller committees. In this paper, I show that the optimal size of committees may be finite even in the absence of free-riding problems induced by costly information acquisition.

My paper differs from most papers on committee decision-making in that members are motivated by career concerns rather than the decision itself. ${ }^{5}$ Accordingly, experts aim to convince outside observers that they are highly competent: Being regarded as highly competent may be desirable because of improved career opportunities (Holmström, 1999). Moreover, experts may value prestige in its own right.

I assume that the arguments presented by the experts are verifiable. ${ }^{6}$ This can be justified by the fact that experts may be able to provide data or detailed explanations that renders their information hard. Conversely, I make the plausible assumption that experts cannot prove that they possess no argument. Hence my framework is one of

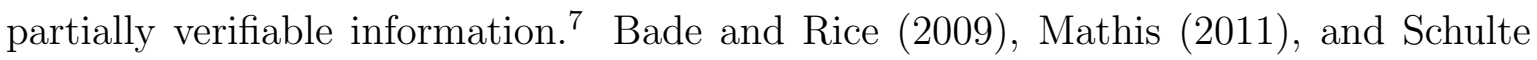

\footnotetext{
${ }^{3}$ Feddersen and Pesendorfer (1998) find that the probability of a wrong decision may increase with the number of committee members if the unanimity rule is used.

${ }^{4}$ See Persico (2004), Martinelli (2007), Koriyama and Szentes (2009), and Gersbach and Hahn (2011) for analyses of committees where members' skills or accuracy of information are endogenous. Gerardi and Yariv (2008) consider the optimal design of a committee with endogenous information acquisition.

${ }^{5}$ Ottaviani and Sørensen (2001, 2006), Visser and Swank (2007), Gersbach and Hahn (2008), and Hahn $(2008,2011)$ also consider committees with experts who have career concerns.

${ }^{6}$ The canonical model of cheap talk and thus non-verifiable information is due to Crawford and Sobel (1982).

${ }^{7}$ For an early paper including partially verifiable information see Shin (1994).
} 
(2010) analyze models of verifiable information and voting. However, in their papers voters are interested in the decision per se, whereas in the present paper experts are only motivated by career concerns.

Hahn (2011) examines sequential information aggregation by committees of experts with partially verifiable information. The present paper differs in several respects from the existing literature and Hahn (2011), in particular. First, I assume here that the quantity of information that can be observed by experts is limited. Consequently, the principal would not make a correct decision with certainty even in the first-best scenario with a very large number of experts. Second, I introduce the novel assumption that the presentation and discussion of an argument in the presence of other committee members and outsiders creates new information. In this sense, the information revealed by the committee is more than just the sum of the pieces of information held by the individual experts. Third, in the present paper I attempt to capture the realistic feature that only one expert can elaborate an argument at a time. Experts in my model decide endogenously whether to compete for the opportunity to raise an argument. ${ }^{8}$

Finally, my paper is related to the winner's curse in common value auctions with private information (see Wilson (1977), Milgrom (1979), and Milgrom and Weber (1982) for seminal contributions and Bolton and Dewatripont (2005), ch. 7, for an overview). The winner's curse describes the observation that a bidder will win the auction exactly in situations where the other agents' signals indicate a lower value of the object than his own signal. Taking this into account, agents bid more cautiously. In my paper, agents who have observed an argument in private are uncertain about its soundness. Releasing the argument would be beneficial to their reputation if it was substantive but harmful if it was inessential. I show that an expert competing with others to present his argument is more likely to be successful in presenting it in situations where it is inessential rather than substantive. This effect makes experts more cautious in raising their arguments.

\footnotetext{
${ }^{8}$ While both in Hahn (2011) and in this paper each expert reveals an argument with positive probability, there are stark differences concerning the optimal size of the committee. In Hahn (2011), very large committees are desirable: In this paper, very large committees may be costly as larger committees make individual experts more reticent.
} 


\section{Model}

I consider a model with a principal, $N \geq 1$ experts, and an additional player, the market. There are two possible states of the world, $\sigma \in\{0,1\}$, which occur with prior probabilities $\left.\pi_{\sigma} \in\right] 0,1\left[\right.$, where $\pi_{0}+\pi_{1}=1$. State 0 is a priori more likely to be correct $\left(\pi_{0}>1 / 2\right)$. Accordingly, I will sometimes refer to state 0 as the prevailing view. The principal faces a decision $d \in\{0,1\}$, whose outcome depends on the state of the world. The principal's utility is

$$
u^{P}= \begin{cases}1 & \text { for } d=\sigma \\ 0 & \text { for } d \neq \sigma,\end{cases}
$$

where 0 and 1 represent convenient normalizations.

Obviously, without additional information the principal would always act in line with the prevailing view, i.e. she would select $d=0$. Before making her decision, the principal consults a committee of experts for evidence against $\sigma=0$ being the state of the world. Each expert may have found an argument that may indicate that the prevailing view is incorrect. For simplicity, I assume that only a single argument may exist at a time. This argument is either substantive $(S)$ or inessential $(I)$. I use $T \in\{S, I\}$ to denote the argument's type (or soundness). Conditional on the state of the world being $\sigma=1$, there is a probability $a(0<a<1)$ of an argument existing and being substantive. In state $\sigma=0$, no substantive argument ever exists. In both cases, $\sigma=1$ and $\sigma=0$, the probability of an argument existing and being inessential is $b(0<b<1-a)$.

A few words about these assumptions are in order. First, the literature so far has focused on the extreme assumption that infinitely many signals can be obtained and that each additional signal may contain information that is not contained in the other signals. By contrast, I make the diametrically opposed assumption that the possible number of arguments is very small. Thus the discovery of duplicate information represents a severe problem. This allows the effects of the boundaries to acquirable knowledge to be studied in the simplest possible case and demonstrates that the usual assumption of infinitely many potentially available signals is not innocuous. Second, I assume that a substantive argument always supports the a priori less likely state. This scenario is more interesting than the one where a substantive argument would 
merely confirm an existing bias and hence would not affect the principal's decision. To simplify the exposition, I focus on the polar case where a substantive argument indicates that $\sigma=1$ is correct with certainty. Third, my assumptions imply that an inessential argument does not affect prior beliefs about the state since an inessential argument occurs with identical probabilities in both states of the world.

There are two types of experts $\tau \in\{H, L\}$, where $H$ stands for a highly efficient expert and $L$ a less competent one. Experts' efficiency types are independent, and the probability of an individual expert being of type $H$ amounts to the commonly known probability $\kappa$ with $0<\kappa<1$. Experts do not have superior information regarding their own efficiency. ${ }^{9}$ Conditional on an argument existing and being of type $T$, the argument is discovered by an expert of type $\tau$ with probability $q_{\tau T}$. I assume that $q_{H S}>q_{L S}$, i.e. a substantive argument can be found more easily by a highly competent expert than a less competent one. In addition, $q_{L I}>q_{H I}$ holds, which implies that less efficient experts are more likely to fall for inessential arguments than their more efficient colleagues. Conditional on the type of argument and the experts' levels of competence, all events of experts finding the argument are independent.

Introducing $\bar{q}_{T}:=\kappa q_{H T}+(1-\kappa) q_{L T} \forall T \in\{I, S\}$, which can be interpreted as the probability of a type $T$ argument being received by an expert of unknown expertise, I can state an important assumption on the $q^{\prime} s$ as

$$
\bar{q}_{S}>\bar{q}_{I}
$$

This condition, which I will assume to hold throughout the paper, has the interpretation that the probability of an argument being found by an expert of unknown expertise, given that the argument is substantive, is higher than the respective probability for an inessential argument. In Section 5.2 I explain how this property can be interpreted as the result of a process of internal reasoning by individual experts.

After observing the argument, each expert can decide to be silent or attempt to release the argument. If more than one expert tries to publish the argument, a single expert is selected from this group to present the argument. Each expert has the identical probability of being selected. The identity of the selected expert becomes commonly known,

\footnotetext{
${ }^{9}$ This assumption is also made in Ottaviani and Sørensen (2001), Visser and Swank (2007), and Hahn (2011). The alternative assumption of known own ability is discussed in Section 5.4.
} 
whereas the identities of the other experts who attempted to release the argument remain unknown.

I offer three approaches to motivate this selection process. First, if experts start to talk during a short period in time (where cognitive limits prevent Bayesian updating), the one who begins will the be the one who presents the argument. ${ }^{10}$ Second, a chairperson might select one of the experts at random. Third, my approach for selecting the expert who has the opportunity to present his argument can be represented by a contest success function that can be characterized completely by the axioms introduced in Skaperdas (1996) and an additional axiom that is natural in the framework studied in this paper. The axiomatic characterization will be provided in Section 5.3.

Experts who have not observed the argument can only remain silent. While experts can always decide not to present the argument and thus to withhold information, they cannot pretend to have an argument when in fact they have none. Hence my model is one of partially verifiable information.

Upon the release of the argument by an expert, the argument's soundness is publicly scrutinized. One way of interpreting this is that there may be a thorough discussion of the argument by all experts and possibly some outsiders. To keep the exposition as simple as possible, I do not model this discussion process explicitly but assume that the type of argument is completely revealed it once has been published by an expert. ${ }^{11}$ While the discussion process completely reveals the type of argument, an individual expert cannot privately assess the soundness of his argument before releasing it. ${ }^{12}$ If no expert attempts to release the argument, the principal does not observe whether an argument exists and, if so, whether it is substantive.

The main motivation for the assumption of the argument's type being observable upon publication is to ensure that, if the argument is released, no further information about the state of the world can be gleaned from the distribution of observations made by

\footnotetext{
${ }^{10}$ Section 5.5 reveals that this paper's findings would not be affected if other experts had the opportunity to speak after the first expert has presented the argument.

${ }^{11}$ An interesting extension to my model would allow for the possibility of this discussion revealing additional information about the experts' levels of competence. Because I want to concentrate on the incentive to release private information, I do not allow for this possibility.

${ }^{12}$ Section 5.2 describes a variant of the model where experts can detect inessential arguments with some positive probability.
} 
other experts. This ensures that the discussion procedure considered in this paper can, in principle, reveal all information about the state that is available to individual agents. ${ }^{13}$

The sole function of the market in my model is to assess the competence of experts after the debate has concluded. This assessment is based on which expert has released the argument (if an argument has been released) and whether the argument was substantive or inessential. The market cannot directly observe the state of the world ${ }^{14}$ or the set of experts who may have attempted to present an argument. However, I assume that the market can observe the existence of the argument and its soundness even if the argument has not been raised by a member of the committee of experts. One possible justification is that the market makes its decision at the final stage of the game, after the principal has taken her decision. The adoption of the principal's decision may reveal additional information and, in particular, the argument that could have been released during the debate. An alternative justification is that the argument may leak out eventually, even if experts try to withhold it. The assumption that the market always knows of the argument's existence and type simplifies the analysis but does not drive my results.

Experts are only interested in a favorable evaluation of their individual competence by the market. Formally, their utility is given by the probability that the market assigns them type $H$. This can be motivated in several ways. More competent experts may receive higher wages (Holmström, 1999) or may have more attractive future career opportunities. Moreover, they may draw utility from the prestige derived from being considered highly competent.

I complete the description of the model by specifying the sequence of events:

1. Nature chooses the state of the world $\sigma \in\{0,1\}$, where $\pi_{\sigma}$ is the probability of $\sigma$ being selected.

2. Nature chooses the levels of competence of individual experts. Each expert is of type $H$ with probability $\kappa$ and of type $L$ otherwise.

\footnotetext{
${ }^{13}$ Suppose, for example, that the type of argument could not be observed perfectly. Then additional information about the state of the world could be inferred from the number of experts who have observed the argument.

${ }^{14}$ However, the observation of a substantive argument immediately reveals that the state of the world is 1 .
} 
3. Nature decides upon the argument's existence and type:

(a) If $\sigma=0$, Nature decides with probability $b$ that an inessential argument exists. With the complementary probability $1-b$, Nature decides that there is no argument.

(b) If $\sigma=1$, an inessential argument exists with probability $b$, as in the previous case where $\sigma=0$. Alternatively, Nature chooses a substantive argument with probability $a(a<1-b)$. With probability $1-a-b$, no argument exists.

4. If there is an argument, then, conditional on the type of the argument $T \in\{S, I\}$ and the expert's competence $\tau \in\{H, L\}$, each expert $i=1, \ldots, N$ will observe the argument with probability $q_{\tau T}$. If there is no argument, all experts will observe nothing.

5. All experts who have observed the argument can simultaneously try to speak.

6. If more than one expert attempts to raise the argument, one of them is selected randomly, where each expert has identical probability of being selected. If no expert attempts to speak, no argument is released.

7. Provided that an argument has been released, the identity of the expert who has successfully released it and the soundness of the argument become commonly known.

8. Based on the information revealed in the debate, the principal makes her decision $d \in\{0,1\}$.

9. The market assesses the probability of each individual expert being of type $H$.

The solution concept is perfect Bayesian Nash equilibrium. In line with the ex-ante symmetry of experts, I focus on symmetric equilibria in (possibly) mixed strategies. I will characterize the set of all equilibria in pure strategies (including those that are asymmetric) in Section 5.1. 


\section{Analysis}

I start the analysis by deriving some general results. In particular, I characterize the principal's optimal behavior and the first-best. Additionally, I prove that it is more difficult to announce a substantive argument successfully over and against an inessential argument.

After these preliminary steps, I focus on the optimal size of committees. As it is impossible to derive an analytical characterization of committee performance for arbitrary committee size, I proceed as follows. I derive the equilibria and the resulting utility of the principal for committees comprising one or two experts analytically. For larger committees, I present numerical results. By letting committee size go to infinity, I then obtain analytical findings about committee performance for very large committees. Next I present the main proposition of the paper (Proposition 1), which proves that finite committees may be optimal. The proof relies on the analytical results about committees comprising one, two or a large number of experts to show that a two-member committee dominates both a single expert and a very large committee in terms of performance. This, in turn, unambiguously implies that the optimal committee size is finite, although it is impossible to find an analytical expression for the optimal size of the committee in general. Finally, I conclude this section by illustrating my previous results with an example.

\subsection{General Results}

It is straightforward to characterize the principal's optimal decision (for a proof see Appendix A):

\section{Lemma 1}

The following strategy of the principal strictly dominates all other strategies:

$$
d= \begin{cases}1 & \text { if a substantive argument has been released } \\ 0 & \text { otherwise }\end{cases}
$$

If all experts attempt to announce their argument with identical probability $\lambda$, provided that they have observed one, and the principal follows strategy (3), her ex-ante expected utility will be

$$
\mathbb{E}\left[u^{P}\right]=\pi_{0}+\pi_{1} a\left(1-\left(1-\bar{q}_{S} \lambda\right)^{N}\right)
$$


Hence, the release of a sound argument induces the principal to go against the prevailing view and to pick $d=1$. In all other cases, the principal sticks to the prevailing view and selects $d=0$.

The expression on the right-hand side of (4) gives the probability of a correct decision. In state $\sigma=0$, which materializes with probability $\pi_{0}$, the correct decision is always taken. Conditional on the state being $\sigma=1$, the principal's decision will only be correct if at least one expert releases a sound argument, which occurs with the probability $a\left(1-\left(1-\bar{q}_{S} \lambda\right)^{N}\right)$.

There are three reasons why the principal may make a wrong decision if $\sigma=1$. First, a substantive argument simply may not exist. Second, such an argument may exist but may not have been noticed by the experts. Third, a substantive argument may exist and may have been observed by at least one expert, but the respective expert(s) may have chosen to keep it secret. The first source of error is unavoidable in my model. The second source can be reduced by enlarging the committee. How the severity of the third source of error is affected by committee size and how this impacts on the optimal committee size is unclear as yet and will be explored in the rest of this paper.

The next lemma characterizes the first-best, i.e. the case where all experts reveal their information truthfully:

\section{Lemma 2}

Suppose the principal could observe the private information of every agent and pursued strategy (3). The principal's utility would increase strictly with committee size and the optimal committee size would be infinity. The principal's expected utility would be

$$
\mathbb{E}\left[u^{P}\right]=\pi_{0}+\pi_{1} a\left(1-\left(1-\bar{q}_{S}\right)^{N}\right)
$$

for a finite committee of size $N$ and

$$
\mathbb{E}\left[u^{P}\right]=\pi_{0}+\pi_{1} a
$$

for $N \rightarrow \infty$.

Note that the probability of a correct decision would be $\pi_{0}+\pi_{1} a$ and thus smaller than one, even if there were infinitely many experts certain of releasing their argument. This 
is due to the fact that error is unavoidable in my model when the state is $\sigma=1$ but no substantive argument exists, which occurs with probability $\pi_{1}(1-a)$.

The next lemma highlights a crucial feature of the model. Loosely speaking, it is easier to publish inessential than substantive arguments. This will have the important consequence that experts may be reluctant to attempt to reveal their arguments because the probability of their revealing an argument successfully is higher in the event of this argument being inessential.

\section{Lemma 3}

Consider an individual expert $i \in\{1, \ldots, N\}$ who has observed an argument. Assume that he attempts to release the argument. Moreover, suppose that all other experts try to release the argument with probability $\lambda \in[0,1]$, provided that they have observed it. Irrespective of the committee size $N$, the probability of expert $i$ being able to present his argument successfully will be weakly higher if the argument is inessential than if it is substantive. This inequality will be strict if $\lambda>0$.

The proof, which is given in Appendix B, relies on the assumption that the probability of a substantive argument being found by an expert of unknown competence exceeds the one for an inessential argument (see (2)). As a consequence, the number of experts who are endowed with an argument will be low if the argument is inessential compared to when it is substantive. This makes it more difficult to present substantive arguments than inessential ones, which constitutes the winner's curse in my model.

To rule out the existence of uninteresting equilibria where all experts never release their arguments, irrespective of the size of the committee, it will be useful to introduce the following variable:

$$
\rho:=\frac{b\left(\bar{q}_{I}-q_{H I}\right)}{\pi_{1} a\left(q_{H S}-\bar{q}_{S}\right)}
$$

In Appendix C, I prove

\section{Lemma 4}

There will be no perfect Bayesian Nash equilibrium in which all experts withhold information with certainty iff

$$
\rho<1 \text {. }
$$


I will assume henceforth that (8) holds. This condition has the following interpretation. It can be easily shown that it is equivalent to

$$
\frac{\kappa\left(\pi_{1} a q_{H S}+b q_{H I}\right)}{\pi_{1} a \bar{q}_{S}+b \bar{q}_{I}}>\kappa .
$$

The right-hand side gives the probability that an expert attaches to the event of his being highly efficient, before he has the opportunity to observe an argument. The lefthand side gives the same probability, after the expert has observed an argument. Thus condition (8) ensures that observing an argument makes the expert more confident in his competence.

\subsection{One or Two Experts}

Having derived preliminary results that hold for arbitrary committee size, I now focus on committees of one or two experts. In these cases, it is possible to solve the model analytically.

\section{Lemma 5}

If the committee consists of one or two experts $(N \in\{1,2\})$, exactly one perfect Bayesian Nash equilibrium will exist. In this equilibrium, experts will attempt to announce their arguments with certainty if they observe an argument. The principal will adopt the strategy described in Lemma 1. In equilibrium, the principal's expected utility will be

$$
\mathbb{E}\left[u^{P}\right]= \begin{cases}\pi_{0}+\pi_{1} a \bar{q}_{S} & \text { for } N=1 \\ \pi_{0}+\pi_{1} a \bar{q}_{S}\left(2-\bar{q}_{S}\right) & \text { for } N=2\end{cases}
$$

I obtain as a corollary that two experts are always preferable to one. Therefore the optimal committee size is always strictly larger than one.

\subsection{Numerical Results}

In this section, I analyze the model for committees with more than two members. As it is impossible to obtain analytical results for arbitrary committee sizes, I rely on numerical computations. I start by establishing the uniqueness of equilibrium (for details see Appendix E). 


\section{Numerical Finding 1}

If

$$
\rho>\frac{\bar{q}_{I}}{\bar{q}_{S}}
$$

then exactly one perfect Bayesian Nash equilibrium with symmetric strategies of experts will exist.

Next I examine the behavior of an individual expert as a function of the number of committee members (for details see Appendix F).

\section{Numerical Finding 2}

Suppose (10) holds and consider the perfect Bayesian Nash equilibria with symmetric strategies of experts for different values of $N(N \geq 3)$. In these equilibria, the probability $\lambda$ of individual experts trying to release an argument, conditional on having observed one, is a weakly monotonically decreasing function of committee size $N$.

Importantly, experts are getting more reticent as the committee becomes larger. ${ }^{15}$ This follows from the fact that, in large committees, it is comparably unlikely that an expert can successfully announce an argument that is substantive, given that substantive arguments are observed with higher probability than inessential ones (see Condition (2)). By contrast, in very small committees, it is quite probable that an expert is the only expert who has observed an argument at all. In this case, the opportunity to announce the argument ahead of the other committee members is not indicative of the argument being inessential.

\subsection{Large Committees}

Analytical results can be obtained for very large committees. These findings are contained in the following lemma:

\footnotetext{
${ }^{15}$ This finding is reminiscent of Feddersen and Pesendorfer (1997), who show that, even in the absence of costs of information collection, the fraction of informative votes may go to zero as the committee size becomes large.
} 


\section{Lemma 6}

Suppose (10) holds and consider the perfect Bayesian Nash equilibria with symmetric strategies of experts for different values of $N$. Then, for large $N$, the probability of an individual expert trying to speak can be approximated as

$$
\lambda=\frac{\alpha}{N}+\mathcal{O}\left(\frac{1}{N^{2}}\right),
$$

where $\alpha$ is a strictly positive constant that is independent of $N$. For $N \rightarrow \infty$, the principal's expected utility converges to

$$
\mathbb{E}\left[u^{P}\right]=\pi_{0}+\pi_{1} a\left(1-e^{-\bar{q}_{S} \alpha}\right) .
$$

The proof is given in Appendix G. In this appendix, I also specify how $\alpha$ can be computed.

Although the probability of an individual expert attempting to release an argument converges to zero (see (11)), the probability of a substantive argument being announced by some expert converges to a finite value. As a consequence, it is unclear by now whether the optimal committee size is finite. This question is addressed in the following.

\subsection{Optimal Size}

Finally, I am in a position to derive the main finding of the paper.

\section{Proposition 1}

If

$$
\rho>\frac{1-e^{-\bar{q}_{S}}}{1-e^{-\bar{q}_{I}}} \cdot \frac{\bar{q}_{I}}{\bar{q}_{S}}
$$

holds, then

1. the optimal committee size will be finite and strictly larger than one, and

2. a single expert will be preferable to an infinitely large committee.

The proof is given in Appendix H. Importantly, the proof does not rely on the numerical findings used to characterize committees of intermediate size. I show in Appendix I that (8) and (13) can be fulfilled simultaneously. 


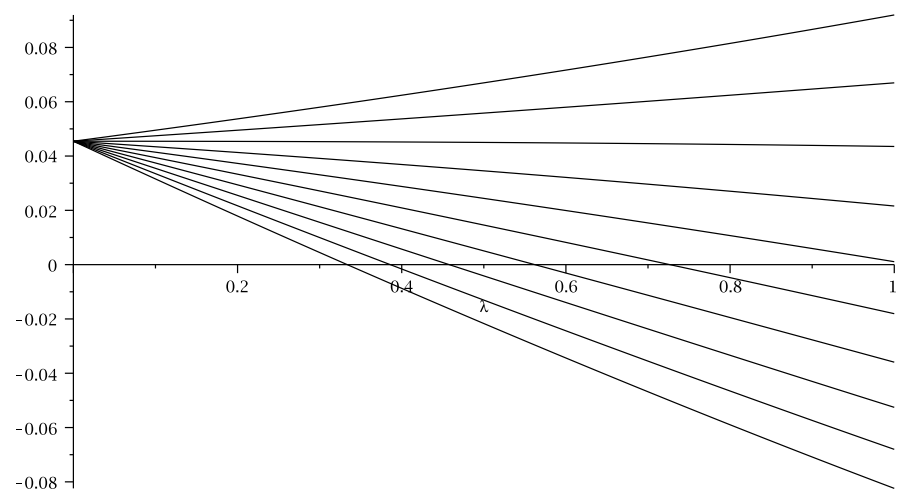

Figure 1: $\Delta$ for $N=1,2, \ldots, 10$ (from top to bottom) as a function of $\lambda$.

While (13) is a sufficient condition for the optimal committee size to be finite, sharper sufficient conditions can be formulated. Because these conditions would be more tedious to derive and the only point of Proposition 1 is to show that a finite committee size can be optimal in my model, I refrain from specifying these conditions. ${ }^{16}$

\subsection{Example}

In this section, I illustrate my findings with an example. I assume $q_{H S}=0.25, \bar{q}_{S}=$ $0.14, \bar{q}_{I}=0.1$, and $q_{H I}=0,{ }^{17}$ which implies $q_{H S}>q_{L S}$ and $q_{L I}>q_{H I}$. Moreover, $a, b$, and $\pi_{1}$ are assumed to be such that $b /\left(a \pi_{1}\right)=1.05$. Obviously, $\bar{q}_{S}>\bar{q}_{I}$, i.e. assumption (2), is fulfilled. With the help of (7), one can easily verify $\rho \approx 0.95$ and thus $\rho<1$ (Assumption (8)). In addition, $\bar{q}_{I} / \bar{q}_{S} \approx 0.71<\rho$, which entails that the condition stated in Numerical Findings 1 and 2 as well as in Lemma 6 holds. Finally, it is easy to check that (13) is violated.

In Appendix $\mathrm{C}$, I define the variable $\Delta$. It is directly proportional to the expected gain in utility achieved by an expert who attempts to release his argument instead of withholding it, given the probability that is commonly assigned to the events of individual experts attempting to release their arguments, $\lambda .{ }^{18}$ So $\Delta>0$ for some $\lambda$

\footnotetext{
${ }^{16}$ For example, one could derive a condition that guarantees that two experts lead to a higher probability of announcing a substantive argument than infinitely many experts.

${ }^{17}$ This constellation would result from $\kappa=1 / 2, q_{H S}=0.25, q_{L S}=0.03, q_{H I}=0$, and $q_{L I}=0.2$, for example.

${ }^{18}$ This means that the market and the principal believe that all experts release their arguments with probability $\lambda$. Moreover, the expert under consideration believes that his colleagues release their arguments with probability $\lambda$.
} 


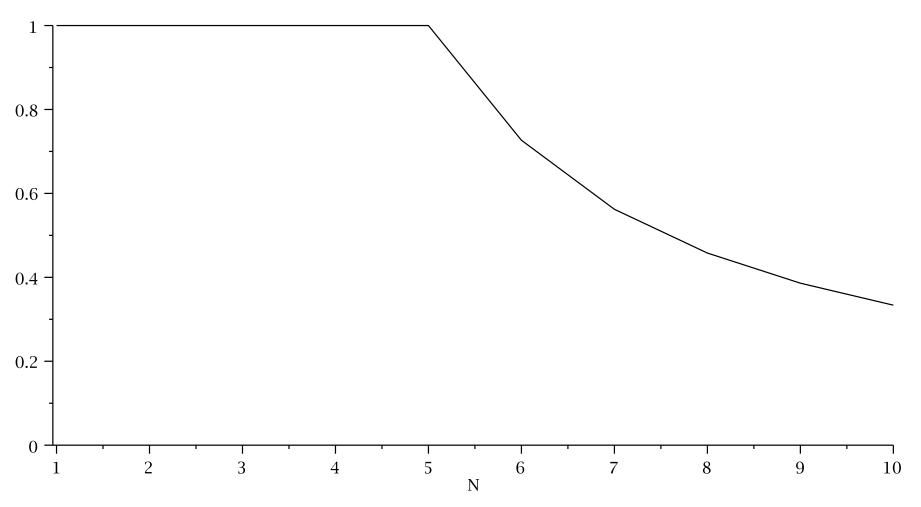

Figure 2: The probability of an individual expert attempting to release his argument $\lambda$ as a function of $N$.

means that it is strictly optimal for an individual expert to compete to announce their argument, given that it is generally believed that all experts follow the mixed strategy characterized by $\lambda$. Conversely, $\Delta<0$ for some $\lambda$ entails that the expert will find it strictly optimal to remain silent.

In Figure 1, I display $\Delta$ as a function of $\lambda$ for different values of $N \geq 1$ and with the value of $N$ increasing from top to bottom. ${ }^{19}$ Several remarks are in order: First, all graphs meet at $\lambda=0$. This is plausible because, if all other experts are certain to be silent, an expert's incentive to reveal his argument will not depend on how many other experts are on the committee. ${ }^{20}$ Second, $\Delta>0$ holds at the point where the graphs meet. This fact follows from Assumption (8), which rules out parameter constellations for which equilibria with $\lambda=0$ exist. Third, $\Delta$ is strictly positive for $N \leq 5$ irrespective of the value of $\lambda$. As a result, a unique equilibrium exists in these cases with $\lambda=1$. For $N>5, \Delta$ is a decreasing function of $\lambda$ and intersects the horizontal axis exactly once. These points correspond to unique equilibria in mixed strategies.

The equilibrium values of $\lambda$ are displayed in Figure 2. While $\lambda$ is constant and equal to one for $N \leq 5$, it decreases in committee size for $N>5$. Thus the larger the committee, the more reticent individual experts become. ${ }^{21}$ As explained before, this

\footnotetext{
${ }^{19}$ It is important to stress that $\Delta$ is not a linear function of $\lambda$, although this may seem to be the case in the figure.

${ }^{20}$ The figure also confirms the observation made in Appendix $\mathrm{D}$ that $\Delta$ increases in $\lambda$ for $N=1$ and $N=2$. Moreover, the fact that $\Delta$ is a decreasing function of $\lambda$ for $N>2$ is in line with results found in Appendix E.

${ }^{21}$ This is reminiscent of Mukhopadhaya (2003), who finds that experts are less likely to pay attention, the larger the committee. In his model, this effect is due to costly information acquisition, which induces free-riding.
} 


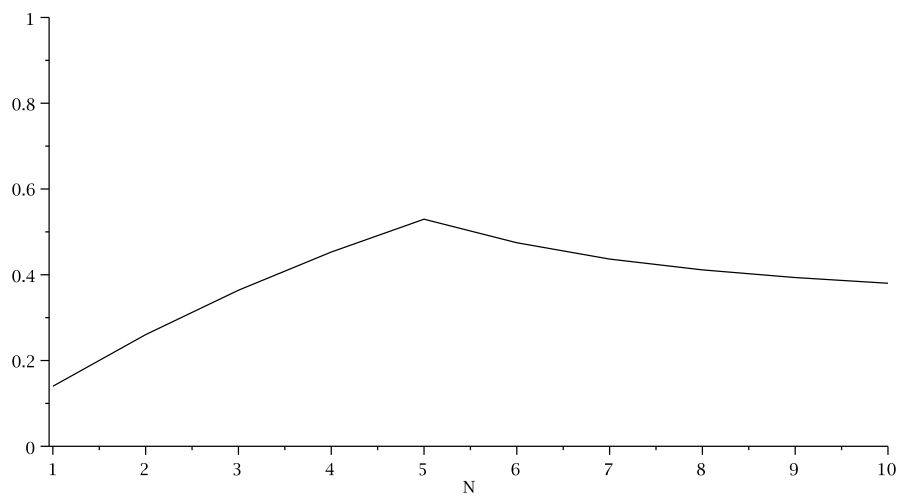

Figure 3: The probability of at least one expert trying to speak, conditional on a substantive argument existing, as a function of $N$.

follows from the fact that experts are more likely to be successful in presenting their argument if this argument is inessential as opposed to substantive. This effect, which makes individual experts more cautious, is particularly strong for large committees. The observation that $\lambda$ is a monotonic function of $N$ confirms Numerical Finding 2 .

Having constructed the equilibrium values of $\lambda$, I can compute the probability of an argument being released by at least one expert, conditional on the argument being substantive. This probability, which can be formally expressed as $\left(1-\left(1-\bar{q}_{S} \lambda\right)^{N}\right)$, directly yields the principal's expected utility (see (4)).

According to Figure 3, the probability under consideration, and thus also the principal's expected utility, increases with committee size for $N \leq 5$. This is an immediate consequence of the observations that $\lambda=1$ for all $N \leq 5$ and that a larger committee size makes it more likely that at least one expert observes any given argument. For $N$ larger than 5, the probability of an individual expert trying to speak, $\lambda$, decreases so strongly that the probability of the argument being raised by one committee member decreases with $N$. As a result, $N=5$ represents the optimal committee size from the principal's perspective.

This is true although the sufficient condition for the optimal committee size to be finite, which is stated in Proposition 1, fails to hold, as can be readily verified (see (13)). Interestingly, a committee of optimal size delivers a substantive argument only with probability 0.53 . This is substantially smaller than in the first-best solution described 
in Lemma 2. In the first-best scenario, a very large committee would be optimal and would reveal a substantive argument with probability one, provided that it existed.

Finally, I would like to note that the probability of a substantive argument being released converges to approximately 0.29 as $N \rightarrow \infty$, which can be computed with (11), (12), and (39). Hence a very large committee is superior to a single expert, who only releases a substantive argument with the probability $\bar{q}_{S}=0.14 .{ }^{22}$

\section{Robustness and Extensions}

In the following, I discuss the robustness of my findings and several extensions to my framework. More specifically, I focus on equilibria in pure strategies, a rationale for the crucial Assumption (2), an axiomatic characterization of the process used to determine which expert may speak, variants of my framework with known own ability, the possibility of more than one expert raising an argument, the existence of more than one argument, and the relationship of the mechanism proposed here to the endogenousinformation-acquisition approach.

\subsection{Equilibria in pure strategies}

In this section, I explain how the full set of equilibria in pure strategies can be constructed. I have already identified the set of symmetric equilibria for different committee sizes $N$. For committees weakly smaller than a threshold level $N^{*}$, these symmetric equilibria exist in pure strategies. In these cases, it can be easily shown that the symmetric equilibria I have characterized represent the only equilibria in pure strategies. For larger $N$, it is straightforward to show that all equilibria in pure strategies involve $N^{*}$ experts releasing their argument with certainty, whereas the remaining experts are silent. Thus, when we consider equilibria in pure strategies, the principal's utility increases with committee size up to $N^{*}$. For larger committees, committee size does not affect her utility. In this sense, committees of finite size $N^{*}$ are weakly optimal. The optimality of finite committees would be strict if I introduced some small costs of hiring experts.

\footnotetext{
${ }^{22}$ Recall that (13), which guarantees the claim of Proposition 1, does not hold for the parameter constellation considered in this example. By contrast, if (13) held, then Proposition 1 could be applied and a single expert would be preferable to a very large committee.
} 


\subsection{Possible foundation of assumption (2)}

In this section, I provide a possible foundation of assumption (2), which has the crucial implication of the winner's curse (see Lemma 3). For this purpose, consider the following sequential procedure, in which experts obtain their arguments.

1. The expert observes the argument with probability $\pi_{\tau} \in(0,1)$, which depends on his competence $\tau \in\{H, L\}$.

2. If the expert has observed an inessential argument, he will recognize its type with probability $\xi_{\tau} \in(0,1)$ and then dismiss it.

This process can be used to provide a foundation for the probabilities $q_{\tau T}$ for all $\tau \in$ $\{H, L\}, T \in\{I, S\}$ by noting that this two-stage process yields:

$$
\begin{array}{ll}
q_{H I}=\pi_{H}\left(1-\xi_{H}\right), & q_{H S}=\pi_{H}, \\
q_{L I}=\pi_{L}\left(1-\xi_{L}\right), & q_{L S}=\pi_{L} .
\end{array}
$$

These equations immediately imply condition (2). Hence (2) can be motivated by a two-step procedure where experts observe arguments in a first step and have some positive probability of identifying inessential arguments in a second step.

\subsection{Axiomatic characterization of the selection process}

In the following I demonstrate that the process which is used to select which expert may speak can be described by a contest success function that can be fully characterized by a set of axioms. ${ }^{23}$

In my model, each expert $i$ can be thought of as choosing one out of two effort levels, $e_{i}=\bar{e}>0$ (attempt to speak) and $e_{i}=0$ (no attempt to speak). The probability of $i$ being selected can be described by a contest success function that maps the set of all possible effort combinations of experts $\{0, \bar{e}\}^{N}$ into combinations of success probabilities $[0,1]^{N}$. I use $\operatorname{csf}^{i}\left(\left\{e_{j}\right\}_{j=1}^{N}\right)$ to denote the probability of $i$ 's success.

Skaperdas (1996) introduces the following axioms. (A1) $\sum_{i=1}^{N} \operatorname{csf}^{i}\left(\left\{e_{j}\right\}_{j=1}^{N}\right)=1$ and $\operatorname{csf}^{i}\left(\left\{e_{j}\right\}_{j=1}^{N}\right) \geq 0$ for all $i=1, \ldots, N$ and all $\left\{e_{j}\right\}_{j=1}^{N} \in\{0, \bar{e}\}^{N}$; if $e_{i}>0$ then

\footnotetext{
${ }^{23}$ See Skaperdas (1996) for a seminal contribution on the axiomatic characterization of contest success functions.
} 
$\operatorname{csf}^{i}\left(\left\{e_{j}\right\}_{j=1}^{N}\right)>0$. (A2) For all $i \in 1, \ldots, N, \operatorname{csf}^{i}\left(\left\{e_{j}\right\}_{j=1}^{N}\right)$ increases in $e_{i}$ and decreases in $e_{k}$ for all $k \neq i$. (A3) For any permutation $\mathcal{P}$ of $(1, \ldots, N)$ (i.e., a bijection $\mathcal{N}:\{1, \ldots, N\} \rightarrow\{1, \ldots, N\})$ we have $\operatorname{csf}^{i}\left(\left\{e_{j}\right\}_{j=1}^{N}\right)=\operatorname{csf}^{\mathcal{P}(i)}\left(e_{\mathcal{P}(1)}, e_{\mathcal{P}(2)}, \ldots, e_{\mathcal{P}(N)}\right) \forall i=$ $1, \ldots, N$.

Axiom (A1) guarantees that $\operatorname{csf}^{i}$ has the properties of a probability distribution and that positive effort results in a positive value of $\operatorname{csf}^{i}$. Moreover, (A2) stipulates that a higher effort should increase one's probability of success and simultaneously lower all others' success probabilities. Finally, (A3) ensures anonymity, i.e. an agent's probability of success does not depend on his identity but only on the effort levels chosen by him and the other experts.

I introduce a fourth axiom that is natural in my model because an expert who does not wish to speak cannot be forced to do so: (A0) If $e_{i}=0$ for some $i$, then $\operatorname{csf}^{i}\left(e_{1}, e_{2}, \ldots, e_{i}, \ldots, e_{N}\right)=0$ for all possible $(N-1)$-dimensional vectors $\left(e_{1}, \ldots, e_{i-1}, e_{i+1}, \ldots, e_{N}\right) \in\{0, \bar{e}\}^{N-1}$ of other experts' effort levels. Axiom (A0) is at odds with axiom (A1) if all experts choose zero effort. Accordingly, I modify (A1) to allow for $\sum_{i} \operatorname{csf}^{i}\left(\left\{e_{j}\right\}_{j=1}^{N}\right)=0$ if $e_{k}=0 \forall k=1, \ldots, N$ but leave it otherwise unchanged. This defines axiom (A1').

It is now immediate to see

\section{Lemma 7}

(A0), (A1'), (A2), and (A3) characterize a unique contest success function. It is given by

$$
\operatorname{csf}^{i}\left(\left\{e_{j}\right\}_{j=1}^{N}\right)= \begin{cases}\frac{e_{i}}{\sum_{j=1}^{N} e_{j}} & \text { if } \sum_{j=1}^{N} e_{j}>0 \\ 0 & \text { if } \sum_{j=1}^{N} e_{j}=0 .\end{cases}
$$

This contest success function is the exact function used in this paper for the probability of experts being able to raise the argument.

\subsection{Known own ability}

A model of a committee of experts where experts know their own ability is more difficult to analyze than the case of unknown own ability, which is considered in this paper. Nevertheless I will attempt to indicate some results that a more thorough treatment of 
this extension could plausibly reveal. In particular, one might conjecture that an expert who knows his efficiency to be high would always release his argument to demonstrate his high ability. In this case, my main finding about finite optimal committee size would break down and infinitely large committees would be optimal.

To address this important issue, note that even if an expert knew his own competence to be high, the expert could not be sure that his argument would be substantial, which follows from $q_{H I}>0$. Moreover, it is natural to guess that the probability of an expert releasing his argument in equilibrium would be weakly higher for experts of type $H$ compared to type $L$. In combination with (2) and $q_{H S}>q_{H I}$, this has the important consequence that the probability of successfully releasing one's argument would be higher for inessential arguments and hence the winner's curse would also occur in a variant of my model with known own ability. ${ }^{24}$ The winner's curse would still be particularly strong in large committees. This, in turn, would make even highly efficient experts reluctant to speak in sufficiently large committees. As a result, in a variant of my model where experts know their types, the optimal committee size is plausibly larger than for unknown own expertise but finite nevertheless.

\subsection{More than one expert may raise the argument}

I have assumed so far that only one expert has the opportunity to present his argument. How would my results be affected if I allowed other experts to present the argument? For this purpose, I extend the basic framework by making the following assumptions. First, once the argument has been released and discussed, it can be presented by all experts. This is plausible because experts should be able to understand the argument and be able to repeat it. Second, after an argument has been presented, other experts have the opportunity to present the same argument. Clearly, under these assumptions, presenting the argument after it has already been released represents cheap talk and does not reveal additional information about the state of the world or the experts' levels of competence. Hence, my results would be unaffected by this extension.

\footnotetext{
${ }^{24}$ This can be easily shown by adapting the proof of Lemma 3 .
} 


\subsection{Different arguments}

Another intriguing extension would involve the possibility of more than one argument being observed. The most straightforward model in which this could be studied would feature a high degree of expert specialization To be more specific, suppose that the problem could be divided into $m$ subtasks ( $m$ finite) and that each subtask involves searching for a particular argument. Then the principal could partition the committee into $m$ disjoint subcommittees, each of which would be assigned one specific subtask. Obviously, my analysis would imply that the optimal size of each subcommittee would be finite, which would entail a finite optimal size of the whole committee.

A model in which each expert could be endowed with more than one argument would be substantially more complex. However, as long as an assumption analogous to (2) holds, a winner's curse would arise, which would be more severe in larger committees than in smaller ones. This might also lead to the optimality of finite committees in such a model.

\subsection{Relationship to the mechanism relying on costly informa- tion acquisition}

As has been explained in Section 2, costly information acquisition and free-riding are at the heart of the alternative approach to explaining why finite committees may be optimal. This alternative approach and the one proposed in this paper have similar implications as both predict that individual experts will contribute less in larger committees. However, the underlying mechanism is different. In the approach based on endogenous information acquisition, agents are actually less well-informed in larger committees and consequently can only release less precise information. According to the explanation advanced in this paper, agents' quality of information is independent of committee size. However, experts tend to release less information when part of larger committees to preserve their reputation. Both channels are not mutually exclusive and, in fact, may reinforce each other. 


\section{Conclusions}

In this paper, I have presented a model of committee decision-making with three novel features. First, experts do not raise arguments simultaneously (like in Visser and Swank (2007)) or in an exogenously given order (like in Hahn (2011)) but compete for the opportunity to speak. This competition shapes incentives to reveal truthfully or withhold private information because winning is informative of an argument's soundness. Second, the amount of information that can be collected by experts is limited. In particular, information may be redundant, as when two experts may observe the same argument. This contrasts with standard analyses of juries where an additional juror is always endowed with a signal that contains information on top of the other signals. Third, the public release of an argument triggers a debate, which, in turn, creates additional information. The combination of these features entails that experts become more reticent when part of a larger committee and thus helps to explain why small committees may be optimal. 


\section{A Proof of Lemma 1}

I note that an arbitrary combination of mixed strategies of experts can be written as $\left(\lambda_{i}\right)_{i=1}^{N} \in[0,1]^{N}$, where $\lambda_{i}$ denotes the probability of expert $i$ attempting to release his argument, provided that he has one. In the following, I consider the three different outcomes of the experts' debate separately and derive the principal's optimal response in each case.

First, the release of a substantive argument indicates $\sigma=1$ with certainty. In line with (1), it is therefore strictly optimal for the principal to choose $d=1$, irrespective of the agents' strategies $\left(\lambda_{i}\right)_{i=1}^{N} \in[0,1]^{N}$.

Second, an inessential argument reveals no information about the state; independently of $\left(\lambda_{i}\right)_{i=1}^{N} \in[0,1]^{N}$. Thus, the posterior probability of $\sigma=0$ being the correct state of the world is equal to the prior $\pi_{0}$, which is strictly larger than $1 / 2$. As a result, selecting $d=0$ is strictly optimal for the principal when an inessential argument has been released. This holds for all combinations of experts' strategies $\left(\lambda_{i}\right)_{i=1}^{N} \in[0,1]^{N}$.

Third, the case where no argument has been released is more intricate. I note that, conditional on the state being $\sigma=0$, there is always a weakly higher probability of no argument being released over and against the situation where $\sigma=1 .^{25}$ This is due to the assumption that each expert has a strictly higher probability of receiving an argument for $\sigma=1$ than for $\sigma=0$ (recall that, for $\sigma=0$, only inessential arguments can arise, whereas for $\sigma=1$ inessential and substantive arguments may occur). As a result, upon no argument being released, the posterior probability of $\sigma=0$ being correct is even higher than the respective prior $\pi_{0}$, which exceeds $1 / 2$ by assumption. This implies that the principal strictly prefers $d=0$.

\section{B Proof of Lemma 3}

Using $\lambda$ for the probability of experts attempting to release their argument, I introduce $\psi_{T n}$ for the probability of exactly $n$ out of $N-1$ experts trying to speak, given the

\footnotetext{
${ }^{25}$ The inequality holds strictly if at least one expert tries to release his argument with strictly positive probability, i.e., the inequality holds strictly for all $\left(\lambda_{i}\right)_{i=1}^{N} \in[0,1]^{N}$ for which $\lambda_{j}>0$ for at least one $j \in\{1, \ldots, N\}$.
} 
argument's type $T \in\{I, S\}$ :

$$
\psi_{T n}:=\left(\begin{array}{c}
N-1 \\
n
\end{array}\right)\left(\bar{q}_{T} \lambda\right)^{n}\left(1-\bar{q}_{T} \lambda\right)^{N-1-n}
$$

Next, consider an individual expert $i \in\{1, \ldots, N\}$. Given that he attempts to speak and that $n$ out of his $N-1$ colleagues also attempt to speak, the probability that he will successfully raise the argument is $1 /(1+n)$. This reflects my assumption that all experts attempting to raise the argument will be successful with equal probability.

Now I can formulate the probability of an expert being able to raise an argument of type $T \in\{I, S\}$ as

$$
\phi_{T}:=\sum_{n=0}^{N-1} \frac{1}{1+n} \cdot \psi_{T n} .
$$

Proving the lemma amounts to showing that

$$
\phi_{I} \geq \phi_{S}
$$

where the inequality is strict if $\lambda>0$. Inequality (16) follows from the observation that, due to (2), the distribution of the number of experts in $\{1, \ldots, N\} \backslash\{i\}$ trying to release an argument, given that $T=S$, first-order stochastically dominates the respective distribution for $T=I$. The fact that $1 /(1+n)$ strictly monotonically decreases in $n$ therefore establishes (16) (see Mas-Colell et al. (1995), p. 195).

\section{Proof of Lemma 4}

In this appendix, I demonstrate that Condition (8) ensures that no equilibrium exists in which all experts withhold their arguments with certainty. For this purpose, I will derive formal expressions for the competence the market assigns to individual experts as well as an expression for the utility gain an expert can achieve by announcing his argument rather than withholding it.

Suppose an expert has successfully announced an argument of type $T \in\{I, S\}$. Which probability will the market assign to the event of this expert being highly competent? The probability of the expert being highly competent and observing an argument of type $T \in\{I, S\}$ is $q_{H T}$. Moreover, the probability of an expert of unknown competency 
observing an argument of type $T$ is $\bar{q}_{T}$. The ratio of these expressions thus gives the probability of the expert being of type $H$. I use $\kappa_{T}, T \in\{I, S\}$, to denote these probabilities:

$$
\begin{aligned}
\kappa_{I} & =\frac{q_{H I}}{\bar{q}_{I}} \kappa \\
\kappa_{S} & =\frac{q_{H S}}{\bar{q}_{S}} \kappa
\end{aligned}
$$

Next I have to specify probabilities of an expert being highly competent for the case where someone else has raised an argument of type $T$, which will be denoted as $\kappa_{o T}$ $T \in\{I, S\}$ ("o" stands for "other"), and for the case where no-one has raised the argument although it existed and was of type $T$. The latter probability will be denoted $\kappa_{\emptyset T}, T \in\{I, S\}$. The different values for $\kappa$ are

$$
\begin{aligned}
\kappa_{O I} & =\frac{1-\psi_{I 0}\left(1-q_{H I} \lambda\right)-q_{H I} \lambda \phi_{I}}{1-\psi_{I 0}\left(1-\bar{q}_{I} \lambda\right)-\bar{q}_{I} \lambda \phi_{I}} \kappa, \\
\kappa_{O S} & =\frac{1-\psi_{S 0}\left(1-q_{H S} \lambda\right)-q_{H S} \lambda \phi_{S}}{1-\psi_{S 0}\left(1-\bar{q}_{S} \lambda\right)-\bar{q}_{S} \lambda \phi_{S}} \kappa, \\
\kappa_{\emptyset S} & =\frac{1-\lambda q_{H S}}{1-\lambda \bar{q}_{S}} \kappa, \\
\kappa_{\emptyset I} & =\frac{1-\lambda q_{H I}}{1-\lambda \bar{q}_{I}} \kappa,
\end{aligned}
$$

where $\lambda$ is the probability of an individual expert attempting to release his argument and $\psi_{T n}$ and $\phi_{T}(T \in\{I, S\})$ are given by (14) and (15). ${ }^{26}$

After these preliminary steps, I am in a position to determine the conditions under which it will be optimal for an expert to attempt to release his argument. If an expert attempts to release his argument, his expected utility will be

$$
\frac{\pi_{1} a \bar{q}_{S}}{\pi_{1} a \bar{q}_{S}+b \bar{q}_{I}}\left[\phi_{S} \kappa_{S}+\left(1-\phi_{S}\right) \kappa_{o S}\right]+\frac{b \bar{q}_{I}}{\pi_{1} a \bar{q}_{S}+b \bar{q}_{I}}\left[\phi_{I} \kappa_{I}+\left(1-\phi_{I}\right) \kappa_{o I}\right],
$$

\footnotetext{
${ }^{26}$ Computing these probabilities is somewhat involved. As an example, I will explain (19) in more detail. Following Bayes' rule, $\kappa_{o I}$, which is the probability of the member under consideration being of type $H$, conditional on "oI," is the ratio of (i) the probability of another member releasing an argument and the member being of type $H$, conditional on an argument of type $I$ existing, and (ii) the respective probability for a member of arbitrary type. Probability (i) is given by $\kappa\left(1-\psi_{I 0}\left(1-q_{H I} \lambda\right)-\right.$ $\left.q_{H I} \lambda \phi_{I}\right)$. This can be seen by noting that there are two different cases where another member may release the inessential argument (constellation "oI"): First, the member under consideration may be competent, have received the argument, and have tried to release it in vain. The respective probability is $\kappa \lambda q_{H I}\left(1-\phi_{I}\right)$. Second, the member may be competent, may not have attempted to speak (either because he did not observe the argument or because he chose to keep it secret), and another expert may have raised the argument. The probability of this happening is $\kappa\left(1-\lambda q_{H I}\right)\left(1-\psi_{I 0}\right)$. The sum of $\kappa \lambda q_{H I}\left(1-\phi_{I}\right)$ and $\kappa\left(1-\lambda q_{H I}\right)\left(1-\psi_{I 0}\right)$ gives probability (i). Probability (ii) can be derived by noting that it is equal to the sum of probability (i) and the respective probability for a member of type $L$.
} 
where I have used the fact that, given the expert's knowledge of an argument existing, the argument is of type $S$ with probability $\left(\pi_{1} a \bar{q}_{S}\right) /\left(\pi_{1} a \bar{q}_{S}+b \bar{q}_{I}\right)$ and of type $I$ with probability $\left(b \bar{q}_{I}\right) /\left(\pi_{1} a \bar{q}_{S}+b \bar{q}_{I}\right)$. If the expert remains silent, his expected utility will be

$$
\frac{\pi_{1} a \bar{q}_{S}}{\pi_{1} a \bar{q}_{S}+b \bar{q}_{I}}\left[\left(1-\psi_{S 0}\right) \kappa_{o S}+\psi_{S 0} \kappa_{\emptyset S}\right]+\frac{b \bar{q}_{I}}{\pi_{1} a \bar{q}_{S}+b \bar{q}_{I}}\left[\left(1-\psi_{I 0}\right) \kappa_{o I}+\psi_{I 0} \kappa_{\emptyset I}\right] .
$$

An expert will find it strictly beneficial to attempt to speak iff (23) is larger than (24), which is equivalent to $\widetilde{\Delta}>0$, where

$$
\begin{aligned}
\widetilde{\Delta}:=\pi_{1} a \bar{q}_{S}\left[\phi _ { S } \left(\kappa_{S}\right.\right. & \left.\left.-\kappa_{o S}\right)+\psi_{S 0}\left(\kappa_{o S}-\kappa_{\emptyset S}\right)\right] \\
& +b \bar{q}_{I}\left[\phi_{I}\left(\kappa_{I}-\kappa_{o I}\right)+\psi_{I 0}\left(\kappa_{o I}-\kappa_{\emptyset I}\right)\right] .
\end{aligned}
$$

If $\widetilde{\Delta}=0$, the expert will be indifferent.

According to (14) and (15), the probability of an individual expert being able to successfully present his argument $(T \in\{I, S\})$ is

$$
\phi_{T}=\sum_{n=0}^{N-1} \frac{1}{1+n}\left(\begin{array}{c}
N-1 \\
n
\end{array}\right)\left(\bar{q}_{T} \lambda\right)^{n}\left(1-\bar{q}_{T} \lambda\right)^{N-1-n} .
$$

Applying $\left(\begin{array}{c}N-1 \\ n\end{array}\right)=\frac{(N-1) !}{n !(N-1-n) !},\left(\begin{array}{c}N \\ n\end{array}\right)=\frac{N !}{(n) !(N-n) !}$, and $\sum_{n=0}^{N}\left(\begin{array}{c}N \\ n\end{array}\right)\left(\bar{q}_{T} \lambda\right)^{n}\left(1-\bar{q}_{T} \lambda\right)^{N-n}=1$, this expression can be rewritten as

$$
\begin{aligned}
\phi_{T} & =\sum_{n=0}^{N-1} \frac{1}{1+n}\left(\begin{array}{c}
N-1 \\
n
\end{array}\right)\left(\bar{q}_{T} \lambda\right)^{n}\left(1-\bar{q}_{T} \lambda\right)^{N-1-n} \\
& =\sum_{n=0}^{N-1} \frac{(N-1) !}{(n+1) !(N-1-n) !}\left(\bar{q}_{T} \lambda\right)^{n}\left(1-\bar{q}_{T} \lambda\right)^{N-1-n} \\
& =\frac{1}{N} \sum_{n=1}^{N} \frac{N !}{n !(N-n) !}\left(\bar{q}_{T} \lambda\right)^{n-1}\left(1-\bar{q}_{T} \lambda\right)^{N-n} \\
& =\frac{1}{N \bar{q}_{T} \lambda} \sum_{n=1}^{N}\left(\begin{array}{c}
N \\
n
\end{array}\right)\left(\bar{q}_{T} \lambda\right)^{n}\left(1-\bar{q}_{T} \lambda\right)^{N-n} \\
& =\frac{1-\left(1-\bar{q}_{T} \lambda\right)^{N}}{N \bar{q}_{T} \lambda} .
\end{aligned}
$$

With the help of this expression for $\phi_{T}, \widetilde{\Delta}$ in (25) can be equivalently stated as

$$
\widetilde{\Delta}=\pi_{1} a\left(q_{H S}-\bar{q}_{S}\right) F\left(\lambda \bar{q}_{S}\right) \kappa-b\left(\bar{q}_{I}-q_{H I}\right) F\left(\lambda \bar{q}_{I}\right) \kappa,
$$


where I have introduced $F(l)$ as

$$
F(l):=\frac{(N-1)(1-l)^{N-2} l^{2}+\left(1-(1-l)^{N-1}\right)^{2}}{(N-1)\left(1-(1-l)^{N}\right) l} .
$$

It will be useful to introduce $\Delta$, which is directly proportional to $\widetilde{\Delta}$, as

$$
\Delta=\frac{F\left(\lambda \bar{q}_{S}\right)}{F\left(\lambda \bar{q}_{I}\right)}-\rho
$$

where, in line with (7), $\rho$ has been defined as

$$
\rho:=\frac{b\left(\bar{q}_{I}-q_{H I}\right)}{\pi_{1} a\left(q_{H S}-\bar{q}_{S}\right)} .
$$

I would like to note that $q_{L I}>q_{H I}$ and $q_{H S}>q_{L S}$ entail $\bar{q}_{I}>q_{H I}$ and $q_{H S}>\bar{q}_{S}$, which, in turn, imply $\rho>0$.

Finally, I am able to compute whether an expert benefits from presenting his argument when all his colleagues withhold information, and thus $\lambda=0$. Note that

$$
\lim _{l \rightarrow 0} F(l)=1 .
$$

Hence $\Delta>0$ is equivalent to

$$
\rho<1,
$$

which is the condition stated in the lemma.

\section{Proof of Lemma 5}

In the following, I examine the cases $N=1$ and $N=2$ separately.

\section{D.1 Single Expert}

First, I analyze the model for a single expert $(N=1)$. For this purpose, it will be useful to note

$$
\lim _{N \rightarrow 1} F(l)=\frac{1}{1-l},
$$

where $F(l)$ has been defined in (28). Inserting (31) into (29) yields

$$
\Delta=\frac{1-\lambda \bar{q}_{I}}{1-\lambda \bar{q}_{S}}-\rho
$$


Condition (2) implies that the expression for $\Delta$ in (32) is a strictly monotonically increasing function of $\lambda$. Moreover, (8) implies that $\Delta>0$ for $\lambda=0$. As a result, $\Delta$ is strictly positive for all admissible values of $\lambda$. As $\Delta$ is proportional to the utility gain achieved by the expert when announcing his argument over and against withholding it, the expert always finds it profitable to release his argument.

\section{D.2 Two Experts}

As a next step, I focus on a committee comprising two experts $(N=2)$. In this case, $F(l)$ (see $(28)$ ) can be written as

$$
\left.F(l)\right|_{N=2}=\frac{1}{1-\frac{1}{2} l} .
$$

This expression can be inserted into (29), which results in

$$
\Delta=\frac{1-\frac{1}{2} \lambda \bar{q}_{I}}{1-\frac{1}{2} \lambda \bar{q}_{S}}-\rho .
$$

As in the scenario with a single expert, $\Delta>0$ for $\lambda=0$ follows from (8). Inserting (33) into (29) reveals that $\Delta$ strictly increases in $\lambda$ due to (2). As a consequence, $\Delta$ is strictly positive for all $\lambda \in[0,1]$. Hence a unique equilibrium exists for $N=2$. In this equilibrium, both experts always attempt to release their arguments.

\section{E Details for Numerical Finding 1}

Uniqueness has been analytically established for $N=1$ and $N=2$ in Lemma 5. For this reason, I consider $N>2$ in the following. Recall that an individual expert will find it attractive to release his argument if $\Delta=\frac{F\left(\lambda \bar{q}_{S}\right)}{F\left(\lambda \bar{q}_{I}\right)}-\rho$ (see (29)) is strictly larger than zero, will prefer to keep his argument secret if $\Delta<0$, and will be indifferent if $\Delta=0$. For a grid spanning the entire range of admissible parameter values and $2<N<100$, I have numerically verified that $\max \left\{\frac{F\left(\lambda \bar{q}_{S}\right)}{F\left(\lambda \bar{q}_{I}\right)}, \bar{q}_{I}\right\}$ is a monotonically decreasing function of $\lambda$ (it is strictly decreasing for $\left.\frac{F\left(\lambda \bar{q}_{S}\right)}{F\left(\lambda \bar{q}_{I}\right)}>\frac{\bar{q}_{I}}{\bar{q}_{S}}\right)$. Together with the observation that $\frac{F\left(\lambda \bar{q}_{S}\right)}{F\left(\lambda \bar{q}_{I}\right)}$ goes to one as $\lambda$ approaches zero (see (30)), this numerical finding implies that, for $1>\rho>\frac{\bar{q}_{I}}{\bar{q}_{S}}, \Delta=0$ can only hold once at most. If $\Delta=0$ holds for one value of $\lambda$, a unique equilibrium exists, which is in mixed strategies. If $\Delta>0$ for all admissible values of $\lambda$, then equilibrium is also unique. In this equilibrium, all experts always attempt to release their arguments $(\lambda=1)$. 


\section{F Details for Numerical Finding 2}

I have numerically demonstrated, for a grid of admissible parameter values with $N \leq$

100, that $\frac{F\left(\lambda \bar{q}_{S}\right)}{F\left(\lambda \bar{q}_{I}\right)}$ decreases in $N$ for arbitrary fixed values of $\lambda$ with $\lambda>0$. Together with the fact that $\frac{F\left(\lambda \bar{q}_{S}\right)}{F\left(\lambda \bar{q}_{I}\right)}$ decreases strictly monotonically as a function of $\lambda$ in the relevant range $\lambda \in[0,1]$, this shows that the value of $\lambda$, implicitly given by $\Delta=0$ decreases in $N$ (if $\Delta>0$, then the equilibrium is in pure strategies with $\lambda=1$ ).

\section{G Proof of Lemma 6}

As a first step, I demonstrate that for $N \rightarrow \infty$, the probability of individual experts attempting to present their argument, $\lambda$, cannot converge to a strictly positive value. For this purpose, I note that for all positive fixed values of $\lambda$

$$
\lim _{N \rightarrow \infty} \frac{F\left(\lambda \bar{q}_{S}\right)}{F\left(\lambda \bar{q}_{I}\right)}=\frac{\bar{q}_{I}}{\bar{q}_{S}}
$$

Consequently, $\Delta$, as defined in (29), converges to

$$
\lim _{N \rightarrow \infty} \Delta=\frac{\bar{q}_{I}}{\bar{q}_{S}}-\rho
$$

This expression is strictly negative due to (10). As a result, the equilibrium value of $\lambda$ cannot converge to a strictly positive value for $N \rightarrow \infty$, as all experts would strictly prefer to withhold their arguments in this case, which contradicts $\lambda>0$.

Inserting $\lambda=\frac{1}{N} \alpha$ into the definition of $\Delta$, i.e. (29), and taking the limit $N \rightarrow \infty$ gives

$$
\lim _{N \rightarrow \infty} \Delta=\frac{1-e^{-\alpha \bar{q}_{S}}}{1-e^{-\alpha \bar{q}_{I}}} \cdot \frac{\bar{q}_{I}}{\bar{q}_{S}}-\rho
$$

where I have utilized

$$
\lim _{N \rightarrow \infty} F\left(\frac{\beta}{N}\right)=\frac{1-e^{-\beta}}{\beta},
$$

which in turn follows from $\lim _{N \rightarrow \infty}(1-\beta / N)^{N}=e^{-\beta}$. Therefore, for $\lambda=\frac{1}{N} \alpha$ and $N \rightarrow \infty, \Delta=0$ is equivalent to

$$
\frac{1-e^{-\alpha \bar{q}_{S}}}{1-e^{-\alpha \bar{q}_{I}}} \cdot \frac{\bar{q}_{I}}{\bar{q}_{S}}=\rho .
$$


Does (39) have a unique admissible solution for $\alpha$ ? The left-hand side of (39) approaches one for small positive values of $\alpha$ and $\bar{q}_{I} / \bar{q}_{S}$ for large values of $\alpha$. Moreover, it decreases monotonically in $\alpha$ since $\bar{q}_{S}>\bar{q}_{I}$. Consequently, (39) has a unique positive solution for $\alpha$ if $\frac{\bar{q}_{I}}{\bar{q}_{S}}<\rho<1$. Hence, for large $N$, the probability $\lambda$ can be approximated as $\lambda=\frac{\alpha}{N}+\mathcal{O}\left(\frac{1}{N^{2}}\right)$, where $\alpha$ is defined implicitly by (39).

\section{H Proof of Proposition 1}

First, I prove the second claim of the proposition. According to this claim, (13) implies that the probability of a single expert leading to a correct decision is higher than the respective probability for a committee comprising infinitely many members. In line with (9), the probability of a correct decision being reached for a single adviser is

$$
\left.\mathbb{E}\left[u^{P}\right]\right|_{N=1}=\pi_{0}+\pi_{1} a \bar{q}_{S} .
$$

For a very large committee, (4) and $\lambda=\alpha / N$ yield

$$
\lim _{N \rightarrow \infty} \mathbb{E}\left[u^{P}\right]=\pi_{0}+\pi_{1} a\left(1-e^{-\bar{q}_{S} \alpha}\right),
$$

where $\alpha$ is the unique positive solution to (39).

In order to prove the second claim stated in the proposition, I have to show that (40) is larger than (41) or, equivalently,

$$
\bar{q}_{S}>1-e^{-\bar{q}_{S}^{\alpha}} .
$$

This condition is always fulfilled if $\alpha<1$. To assess whether $\alpha<1$ actually holds, I examine (39), which implicitly defines $\alpha$, more closely. I have already noted that the left-hand side of (39) approaches one for small positive values of $\alpha$. For $\alpha \rightarrow 0$, it is therefore larger than the right-hand side of (39), i.e. $\rho$ (compare (8)). Moreover, the left-hand side of (39) is equal to $\frac{1-e^{-\bar{q}_{S}}}{1-e^{-\bar{q}_{I}} \bar{q}_{I}}$ for $\alpha=1$. This value is smaller than $\rho$ according to (13). Together with the observation that the left-hand side of (39) decreases monotonically in $\alpha$, these findings imply a unique solution for $\alpha$ with $\alpha<1$. Having demonstrated that a single expert is superior to an infinitely large committee, I have to complete the proof by showing that the optimal committee size is strictly larger than one. For this purpose, I refer to the probability of two experts leading to a correct decision, which is stated in (9). Noting that this probability is strictly higher than the respective probability for a single expert completes the proof. 


\section{Proof that both (8) and (13) can hold at the same time}

To confirm that (8) and (13) can hold at the same time, it is sufficient to show that

$$
\frac{1-e^{-\bar{q}_{S}}}{1-e^{-\bar{q}_{I}}} \cdot \frac{\bar{q}_{I}}{\bar{q}_{S}}<1 .
$$

Inequality (42) follows from the facts that $\left(1-e^{-x}\right) / x$ is a strictly monotonically decreasing function of $x$ and $\bar{q}_{S}>\bar{q}_{I}$. 


\section{References}

David Austen-Smith and Jeffrey S. Banks. Information aggregation, rationality, and the Condorcet Jury Theorem. The American Political Science Review, 90(1):34-45, March 1996.

David Austen-Smith and Timothy J. Feddersen. Information aggregation and communication in committees. Philosophical Transactions of the Royal Society B: Biological Sciences, 364(1518):763-769, 2009.

Sophie Bade and Andrew Rice. Political advocacy with collective decision making. mimeo, 2009.

Ruth Ben-Yashar and Shmuel Nitzan. The invalidity of the Condorcet Jury Theorem under endogenous decisional skills. Economics of Governance, 2(3):243-249, November 2001.

Sven Berg. Condorcet's Jury Theorem, dependency among jurors. Social Choice and Welfare, 10(1):87-95, 1993.

Philip J. Boland. Majority systems and the Condorcet Jury Theorem. The Statistician, 38(3):181-189, 1989.

Patrick Bolton and Mathias Dewatripont. Contract Theory. Cambridge, MA: MIT Press, 2005.

M. J. A. N. de Caritat Condorcet. An essay on the application of analysis to the probability of decisions rendered by a plurality of votes. In Iain McLean and Arnold B. Urken, editors, Classics of Social Choice. Ann Arbor: University of Michigan Press, 1995, 1785.

Vincent P. Crawford and Joel Sobel. Strategic information transmission. Econometrica, 50(6):1431-1451, November 1982.

Timothy Feddersen and Wolfgang Pesendorfer. Voting behavior and information aggregation in elections with private information. Econometrica, 65(5):1029-1058, September 1997. 
Timothy Feddersen and Wolfgang Pesendorfer. Convicting the innocent: The inferiority of unanimous jury verdicts under strategic voting. American Political Science Review, 92(1):23-35, March 1998.

Dino Gerardi and Leeat Yariv. Costly expertise. The American Economic Review, 98 (2):187-193, May 2008.

Kerstin Gerling, Hans Peter Grüner, Alexandra Kiel, and Elisabeth Schulte. Information acquisition and decision making in committees: A survey. European Journal of Political Economy, 21(3):563-597, September 2005.

Hans Gersbach and Volker Hahn. Should the individual voting records of central-bank council members be published? Social Choice and Welfare, 30(4):655-683, May 2008.

Hans Gersbach and Volker Hahn. Information acquisition and transparency in committees. International Journal of Game Theory, 41(2):427-453, July 2011. forthcoming.

Volker Hahn. Committees, sequential voting and transparency. Mathematical Social Sciences, 63(3):366-385, November 2008.

Volker Hahn. Sequential aggregation of verifiable information. Journal of Public Economics, 95(11-12):1447-1454, December 2011.

Bengt Holmström. Managerial incentive problems: A dynamic perspective. The Review of Economic Studies, 66(1):169-182, January 1999.

Irving L. Janis. Victims of Groupthink: A Psychological Study of Foreign-Policy Decisions and Fiascoes. Oxford, England: Houghton Mifflin, 1972.

Yukio Koriyama and Balazs Szentes. A resurrection of the Condorcet Jury Theorem. Theoretical Economics, 4(2):227-252, June 2009.

Krishna K. Ladha. The Condorcet Jury Theorem, free speech, and correlated votes. American Journal of Political Science, 36(3):617-634, April 1992.

Hao Li and Wing Suen. Decision-making in committees. Canadian Journal of Economics, 42(2):359-392, 2009.

César Martinelli. Rational ignorance and voting behavior. International Journal of Game Theory, 35(3):315-335, October 2007. 
Andreu Mas-Colell, Michael D. Whinston, and Jerry R. Green. Microeconomic Theory. Oxford University Press, New York, 1995.

Jérôme Mathis. Deliberation with evidence. American Political Science Review, 105 (3):516-529, August 2011.

Paul R. Milgrom. A convergence theorem for competitive bidding with differential information. Econometrica, 47(3):679-688, May 1979.

Paul R. Milgrom and Robert James Weber. A theory of auctions and competitive bidding. Econometrica, 50(5):1089-1122, September 1982.

Kaushik Mukhopadhaya. Jury size and the free rider problem. The Journal of Law, Economics, and Organization, 19(1):24-44, 2003.

Marco Ottaviani and Peter Norman Sørensen. Information aggregation in debate: Who should speak first? Journal of Public Economics, 81(3):393-421, September 2001.

Marco Ottaviani and Peter Norman Sørensen. Reputational cheap talk. The RAND Journal of Economics, 37(1):155-175, spring 2006.

Nicola Persico. Committee design with endogenous information. Review of Economic Studies, 71(246):165-191, January 2004.

Elisabeth Schulte. Information aggregation and preference heterogeneity in committees. Theory and Decision, 69(1):97-118, 2010.

Hyun Song Shin. News management and the value of firms. The RAND Journal of Economics, 25(1):58-71, Spring 1994.

Stergios Skaperdas. Contest success functions. Economic Theory, 7(2):283-290, June 1996.

Bauke Visser and Otto Swank. On committees of experts. Quarterly Journal of Economics, 122(1):337-372, February 2007.

Robert Wilson. A bidding model of perfect competition. The Review of Economic Studies, 44(3):511-518, October 1977.

Jörgen Wit. Rational choice and the Condorcet Jury Theorem. Games and Economic Behavior, 22(2):364-376, February 1998. 